\title{
Uso de diários reflexivos no aprendizado de médicos residentes em hospital oncológico no Pará
}

\author{
Reflective diary use in the learning for physician residents at an oncological hospital in \\ Pará
}
Uso del diario reflectante en el aprendizaje los doctors residentes en el hospital oncológico del Pará

\begin{abstract}
Fernanda de Nazaré Cardoso dos Santos Cordeiro ${ }^{1,2 *}$, Herbert Paulino Cordeiro ${ }^{2}$, Brenda Nazaré Gomes Andriolo², José Antonio Cordero da Silva².
\end{abstract}

\begin{abstract}
RESUMO
Objetivo: Descrever a percepção dos médicos residentes quanto ao processo de avaliação no contexto de aprendizado e descrever as características éticas e de seus relacionamentos no contexto da implementação do Diário Reflexivo. Métodos: Estudo observacional, transversal, qualitativo de outubro a dezembro de 2018 por meio do preenchimento de questionários sobre o cotidiano dos médicos residentes e a forma como são avaliados e como gostariam de ser avaliados. Resultados: Foi observado que $68,4 \%$ dos participantes não está satisfeita com a forma como é avaliada e gostaria que fossem utilizadas ferramentas mais inovadoras. Dentre as características dos residentes evidenciou-se que a maioria, 73,7\%, tem formação teórica e se considera humanista. A maioria dos participantes mostrou que anseia por métodos avaliativos mais próximos da realidade e que auxiliem na construção de competências para a vida profissional. Conclusão: O Diário Reflexivo permite o acompanhamento do processo de ensino-aprendizagem e motiva uma contínua reflexão podendo ser usado em diferentes cenários de aprendizagem, emancipando o aluno.
\end{abstract}

Palavras-chave: Residência médica, Avaliação educacional, Aprendizagem, Diário.

\section{ABSTRACT}

Objective: Describe the perception of resident physicians regarding the assessment process in the learning context and to describe the ethical and relationship characteristics of resident physicians in the context of the implementation of the Reflective Diary. Methods: An observational, cross-sectional, qualitative study was conducted from October to December 2018 by filling in questionnaires with questions about the daily lives of resident physicians and how they are evaluated and how they would like to be evaluated. Results: It was observed that $68.4 \%$ of the participants are not satisfied with the forms of evaluation and would like more innovative tools to be used. Among the characteristics of the residents, it was evident that the majority, $73.7 \%$, have a theoretical background and consider themselves humanizeds. Most participants showed that they yearn for evaluative methods that are closer to reality and that help to build competences for professional life. Conclusion: The Reflective Diary assist the monitoring of the teaching-learning process and motivates a continuous reflection that can be used in different learning scenarios, emancipating the students.

Key words: Medical residency, Educational assessment, Learning, Diary.

\section{RESUMEN}

Objetivo: Describir la percepción de los médicos residentes a cerca respecto al proceso de evaluación en el contexto de aprendizaje y describir las características éticas y de relación de los médicos residentes en el contexto de la implementación del Diario reflexivo. Métodos: estudio observacional, transversal y cualitativo, de octubre a diciembre de 2018, completando cuestionarios con preguntas sobre la vida cotidiana de los médicos residentes y cómo se evalúan y cómo les gustaría ser evaluados. Resultados: Se observó que el $68,4 \%$ de los participantes no está satisfecho con la forma en que se evalúa y desea que se utilicen herramientas más innovadoras. Entre las características de los residentes, era evidente que la mayoría, el

1 Universidade do Estado do Pará (UEPA), Belém - PA. *E-mail: fernandancscordeiro@ hotmail.com

${ }^{2}$ Centro Universitário Metropolitano da Amazônia (UNIFAMAZ), Belém - PA.

SUBMETIDO EM: 10/2019 
73.7\%, tenía antecedentes teóricos y se consideraba humanista. La mayoría de los participantes mostraron que anhelan métodos de evaluación más cercanos a la realidad y que ayuden a construir competencias para la vida profesional. Conclusión: El Diario reflexivo permite el seguimiento del proceso de enseñanzaaprendizaje y motiva una reflexión continua que puede utilizarse en diferentes escenarios de aprendizaje, emancipando al alumno.

Palabras-clave: Residencia médica, evaluación educativa, aprendizaje, diario.

\section{INTRODUÇÃO}

A avaliação do processo educacional tem como finalidade emitir juízo guiando as condutas e deve ser realizada a qualquer momento, em qualquer nível e não apenas no final de uma disciplina, módulo ou curso para evitar abordagem pontual, não ser estressante, estigmatizadora ou punitiva, mas sim ajudar no binômio ensino-aprendizagem como um todo, aliando-se, portanto, a formação profissional, oferecendo diretrizes para definir prioridades e tomar as decisões necessárias (CARDOSO DAS, et al., 2015).

O modelo educativo atual é baseado na formação por competências e no centro dessas ações está o aluno, requerendo apropriação crítica do conhecimento (COTTA RMM, et al., 2016). Este tipo de aprendizagem permite ao estudante refletir sobre opções, ter feedback imediato e individualizado, estimulando a progressão com melhora significativa, além do desenvolvimento de habilidades relacionadas com tomada de decisões melhorando também a equipe docente e favorecendo a integração dos conhecimentos aplicados a contextos reais (FIGUEROA C, et al., 2015; COTTA RMM, et al., 2015)

Com a introdução de metodologias e instrumentos pedagógicos ativos de ensino, aprendizagem e avaliação oportunizam-se ao aluno autonomia, criatividade e responsabilidade, aprendendo a buscar soluções e resolver problemas profissionais, dando protagonismo ao estudante, valorizando a aprendizagem significativa e reflexiva, compartilhando saberes e experiências entre professores e estudantes (CARDOSO DAS, et al., 2015; FORTE FDS, et al., 2015).

O Diário Reflexivo, ou portfólio reflexivo, vêm sendo apontado como uma importante contribuição para tornar o processo de avaliação mais eficaz e torna-se uma alternativa supostamente eficaz para obtenção de avaliação formativa de médicos Residentes, objetivando reduzir a deficiência que esta avaliação apresenta nos Programas de Residência médica (O'BRIEN CL, et al., 2016; COTTA RMM, et al., 2015).

A Residência Médica é uma pós-graduação em serviço e, em muitos serviços, sempre foi baseado em algumas aulas teóricas expositivas que se repetiam a cada período, demonstrando uma falta de conteúdo programático mínimo a ser desenvolvido, praticamente sem inovações. A forma de avaliação utilizada acabava limitando-se a uma prova de conhecimentos, considerando-se um programa pedagógico insuficiente para alcançar uma aprendizagem adequada, no sentido de contribuir para a formação de um perfil profissional com as características recomendadas (SOUZA RGS, 2012).

Em um processo de ensino-aprendizagem que objetiva formar profissionais médicos críticos, reflexivos e preparados para atender as demandas da comunidade em que está inserido, deve-se planejar um processo de avaliação adequado e sistematizado que permita o alcance de objetivos pré-estabelecidos como adequados para este fim, pelos médicos residentes. Espera-se, com a construção do Diário Reflexivo, definir lacunas de conhecimento, demonstrar qualidades e conseguir soluções para suprir as necessidades de um médico em formação. Faz-se necessário trazer para o médico residente a responsabilidade compartilhada sobre sua própria aprendizagem, estimulando o pensamento crítico, a criatividade, autoavaliação, articulação, solução de problemas, possibilitando autonomia intelectual, permitindo a análise do progresso do discente, com consequente melhoria das atitudes em situações práticas em favor da comunidade. O portfólio reflexivo torna-se um instrumento importante para agregar todas estas qualidades importantes na formação de um médico residente (CESARIO JB, et al., 2016; BERNARDI MC, et al., 2015).

Devido à grande quantidade de benefícios demonstrados e buscando conhecer um pouco mais dos Programas de Residência médica do estado do Pará objetiva-se com este estudo descrever a percepção dos médicos residentes quanto ao processo de avaliação no contexto de aprendizado de forma prática e 
sistematizada durante o ensino-aprendizagem, além de descrever as suas características quanto a aspectos éticos, de humanização e características de relacionamento com a equipe, pacientes e entre os próprios residentes, bem como o tipo de avaliação a que são submetidos dentro de cada programa de Residência Médica.

\section{MÉTODOS}

Foi realizado um estudo transversal, observacional, descritivo, exploratório, com abordagem qualitativa em no Hospital Oncológico de Referência do estado do Pará no período de outubro a dezembro de 2018. Os participantes da pesquisa foram os médicos residentes do hospital regularmente matriculados no Programa de Residência médica nas especialidades clínicas que aceitaram participar da pesquisa, divididos nos Programas de Clínica Médica, Neurologia, Oncologia Clínica e Hematologia e Hemoterapia. Apenas os residentes clínicos foram selecionados para o estudo por questão de proximidade entre os métodos de avaliação. Foram excluídos os residentes que não estavam matriculados regularmente, os que não aceitaram participar e os médicos residentes de especialidades não clínicas.

O projeto foi encaminhado à Comissão Científica do Hospital, e, após sua aprovação, para o Comitê de Ética em Pesquisa (CEP), onde obteve sua aprovação sob o parecer número 2.701.996, seguindo as normas estabelecidas na Resolução 466/2012 sobre pesquisa em seres humanos e obedecendo aos preceitos éticos da Declaração de Helsinque e do Código de Nuremberg. Após a aprovação do CEP, os residentes que aceitaram participar da pesquisa assinaram o Termo de consentimento livre e esclarecido (TCLE) contendo as informações necessárias acerca da pesquisa.

Os participantes receberam questionários (Apêndice A - Disponível no arquivo suplementar) aplicados pela pesquisadora principal sobre questões norteadoras do cotidiano do Programa de Residência Médica, divididos em três partes: $O$ questionário 1 abordou sobre o motivo pela escolha da especialidade e daquele como hospital para realizar a residência médica. O questionário 2 abordou questões sobre o relacionamento dos residentes com os pacientes e o questionário 3 continha questões acerca da relação dos participantes com a equipe multiprofissional e os outros residentes, além de perguntas sobre o método de avaliação a que são submetidos. Os Residentes foram abordados no local de atuação profissional, após contato prévio, a partir da disponibilidade dos e-mails e telefone pela secretária do Comissão de Residência Médica (COREME) do local. As perguntas contidas no questionário buscaram relatar sobre o cotidiano nos cenários de aprendizagem, mediante o enquadramento teórico que fundamenta o estudo. O questionário também continha perguntas abertas acerca do processo de avaliação e grau de satisfação de cada programa. Durante o preenchimento do questionário os participantes fizeram relatos espontâneos para a autora acerca das vivências exploradas nas questões. Serviu também para armazenar as informações relacionadas à comunidade e a sua prática diária, possibilitando a reflexão como fonte de informações acerca das necessidades da aprendizagem.

Para interpretação dos dados das perguntas abertas foi realizada a análise de conteúdo baseada nos critérios de Bardin L (2011), adotando-se categorias pré-definidas de acordo com os objetivos da pesquisa e após intensa revisão da literatura atual. Esta análise foi dividida em três etapas: a pré-análise, onde se organizou o material após uma leitura inicial, exploração do material com categorias e a última etapa foi a do tratamento dos resultados, com a interpretação das informações a partir de uma análise crítica e reflexiva baseada no referencial teórico.

\section{RESULTADOS E DISCUSSÃO}

O número total de residentes de especialidades clínicas no local de estudo foi de 22. Apenas três residentes não participaram, pois estavam em módulos fora da cidade de Belém e não puderam responder ao questionário e nem assinar o TCLE no período estudado. As respostas dadas nos questionários foram avaliadas conjuntamente as declarações dos médicos residentes participantes, pois, a maioria destas declarações foram feitas durante o preenchimento dos questionários (Tabelas 1 e 2). 


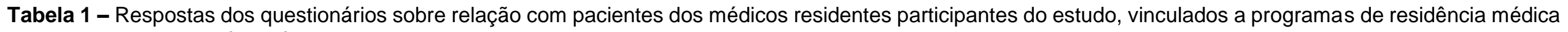
em um Hospital no Pará, Belém - PA, 2019.

PERGUNTA

$\mathrm{N}$ de respostas

1) Quando o paciente não tem condições financeiras para comparecer a próxima consulta você:

Pede para conversar com assistente social para resolver a questão.

Eu mesmo irei resolver dando dinheiro para o paciente.

2) Como você vê a situação em que um paciente demora muitas semanas para realizar um procedimento / internação / consulta:

Infelizmente o SUS é assim e necessita de melhorias.

A situação tem que ser resolvida o mais rápido possível, nem que eu mesmo tenha que fazer isso.

3) O paciente que você atendeu não consegue realizar os exames solicitados:

Infelizmente o SUS é assim e necessita de melhorias.

A situação tem que ser resolvida o mais rápido possível, nem que eu mesmo tenha que fazer isso.

4) O que você acha da situação de muitos pacientes que tem que chegar de madrugada ou até mesmo dormir na fila para conseguir uma consulta ou um exame?

Infelizmente o SUS é assim e necessita de melhorias.

5) Quando o paciente precisa de alguma medicação e/ou material que não normalmente disponibilizado pelo SUS:

Você explica a situação para o paciente e diz que não há o que fazer.

Tenta reverter pedindo auxílio para assistente social e diretoria - solicito compra na farmácia. 
6) Você sente que cria vínculos com os pacientes:

Sempre.

Quando são da minha idade.

$14(73,6 \%)$

Quando lembram alguém da família.

$4(21,05 \%)$

Nunca.

$0(0 \%)$

TOTAL

$1(5,2 \%)$

7) Você decide tratar a doença ou o paciente:

Sempre a doença para não criar vínculos e sofrer.

$0(0 \%)$

O paciente, porque é o que ele merece, independente do que eu sinta ou ache.

$19(100 \%)$

TOTAL

19

8) Qual a importância da qualidade de vida do paciente:

Fundamental, mesmo que não possa tratar sua doença.

$19(100 \%)$

Penso nela, porém me preocupo mais com seu tratamento.

$0(0 \%)$

TOTAL

9) Você indica outras atividades aliadas ao tratamento clínico-cirúrgico para seu paciente:

Sempre.

Somente quando há uma comprovação cientifica de seu benefício.

Somente a pedido do próprio paciente.

TOTAL

\section{0) Sobre terminalidade da vida:}

Busco sempre a cura em primeiro lugar quando o paciente é jovem, pois esta é a função do médico.

Minha opção busca os cuidados paliativos independentemente da idade, buscando a qualidade de vida.

Faço o que meus chefes e a família e o paciente orientam.

TOTAL

Fonte: Cordeiro FNCS, et al., 2019. 
Tabela 2 - Respostas dos questionários sobre relação com equipe dos médicos residentes participantes do estudo, vinculados a programas de residência médica em um Hospital do Pará, Belém - PA, 2019.

\begin{tabular}{|c|c|}
\hline PERGUNTA & $\mathrm{N}$ de respostas \\
\hline \multicolumn{2}{|l|}{ 1) Qual sua relação com os outros residentes? } \\
\hline Muito boa, são meus amigos. & $11(57,8 \%)$ \\
\hline Boa, são colegas de trabalho. & $8(42,1 \%)$ \\
\hline Ruim & $0(0 \%)$ \\
\hline Indiferente. & $0(0 \%)$ \\
\hline TOTAL & 19 \\
\hline \multicolumn{2}{|l|}{ 2) Qual sua relação com os chefes? } \\
\hline Muito boa, são mestres e me ensinam tudo. & $12(63,1 \%)$ \\
\hline Boa, me ensinam o que eu devo aprender. & $7(36,8 \%)$ \\
\hline Regular, somos distantes, sem relação. & $0(0 \%)$ \\
\hline Ruim. & $0(0 \%)$ \\
\hline TOTAL & 19 \\
\hline \multicolumn{2}{|l|}{ 3) Você considera que tem qualidade de vida como médico residente? } \\
\hline Tenho qualidade de vida de qualquer médico, me sinto cansado, mas consigo estudar. & $3(15,7 \%)$ \\
\hline Queria ter mais qualidade de vida, ter menos plantões. & $4(21,05 \%)$ \\
\hline Tenho qualidade de vida de qualquer residente, mas me sinto cansado demais e consigo estudar. & $9(47,3 \%)$ \\
\hline Me sinto muito cansado e não consigo estudar. & $3(15,7 \%)$ \\
\hline TOTAL & 19 \\
\hline
\end{tabular}

REAS/EJCH | Vol.Sup.n.43 | e2174 | DOI: https://doi.org/10.25248/reas.e2174.2020 Página 6 de 11 


\section{4) Sua relação com a equipe multiprofissional:}

É ótima, sem eles não sou nada.

É boa, me ajudam, cada um na sua função.

É necessário em alguns momentos, consigo realizar minhas funções mesmo sem eles.

TOTAL

5) Sua relação com discentes/internos:

Ótima, me ajudam muito.

Boa, apesar de as vezes atrapalharem.

Não precisaria deles.

TOTAL

6) Você ajuda os discentes/internos:

Sempre que me pedem.

Sempre que acho necessário.

$\mathrm{Na}$ maioria das vezes não tenho tempo para isso.

TOTAL

$3(15,7 \%)$

12

$4(21,05 \%)$

19

$7(37,8 \%)$

$11(57,8 \%)$

$1(5,2 \%)$

19

$17(89,4 \%)$

$1(5,2 \%)$

$1(5,2 \%)$

19

Fonte: Cordeiro FNCS, et al., 2019. 
A Tabela 1 mostra as respostas dadas pelos Residentes sobre sua relação com os pacientes atendidos durante seu período de aprendizado. As perguntas versavam sobre dificuldades financeiras destes pacientes e as dificuldades apresentadas quanto a marcação de exames, prescrição de medicamentos e internação hospitalar. A maioria dos residentes mostrou-se preocupado, demonstrando empatia em relação a estes conflitos que acabam por trazer mais tribulações e angústias absorvidas pelos médicos residentes.

Segundo Webb TP, et al. (2014) o relacionamento de médicos e seus pacientes pode ser aperfeiçoado através do uso de tecnologias e ferramentas que levem ao domínio de competências, otimizando não só a aprendizagem, como também o tratamento dedicado aos pacientes que se encontram em posição vulnerável, especialmente no cenário da realização deste estudo, ou seja, um ambiente real dentro do contexto da Saúde Pública. (STELET BP, et al., 2017)

A Tabela 2 mostra as respostas dos Residentes acerca do relacionamento destes participantes com os outros residentes e equipe multiprofissional, incluindo seus chefes, enfermagem e internos de Medicina. As questões nesse momento abordaram sobe coleguismo, qualidade de vida e rotina das atividades diárias. Podemos notar que grande parte das respostas demonstra boa relação entre todos, com cada um realizando o que lhe compete de função.

É importante notar que a relação entre colegas de profissões da área de saúde se faz necessária para o bem-estar de todos, principalmente do paciente, que se torna mais bem assistido. $O$ apoio torna o residente mais motivado e aumenta sua confiança no trabalho e no aprendizado no contexto do mundo real. $\mathrm{O}$ dia-adia e o ambiente de aprendizagem se tornam mais fáceis com evidências de melhorias e progresso real. (CHANG C, et al., 2011). Quando perguntados sobre a sua relação com os pacientes os residentes revelaram que, muitas vezes, eles mesmos resolveriam os problemas relacionados ou não a seu estado patológico, se pudesse, já que, dentro da realidade vivenciada no Sistema Único de Saúde (SUS), há morosidade na resolução dos problemas. Alguns relataram que, por vezes, se sentem culpados por não conseguirem ter sucesso nessa empreitada:

"Já dei dinheiro para comprar remédios que não tinham na farmácia do Hospital, hoje faço menos, devido o dinheiro estar contado para mim também".

A maioria dos estudantes revelou sempre criar vínculos com os pacientes, buscando tratar o paciente e não sua doença, demonstrando que se apegam as pessoas muito mais do que deveriam:

"Dou meu número de telefone para os pacientes e familiares para ajudar no que puder."

Os residentes também revelaram achar fundamental a qualidade de vida de cada um dentro do contexto em que vive, ou seja, de estudante num programa de pós-graduação em que aprende fazendo não só competências relacionadas a conhecimentos dentro da Medicina, mas também competências cognitivas e metacognitivas levando ao crescimento pessoal e profissional.

Quando se tratou da relação com equipe a maioria dos médicos relatou ter relação muito boa com os outros residentes, até mesmo de outras especialidades, e com os chefes de seu programa. A relação com internos e equipe multiprofissional foi definida, pela maioria, como sendo boa, o que é importante dentro do processo de ensino-aprendizagem, pois facilita a construção de um profissional que sabe trabalhar em equipe, pois sabe da importância de cada um. Em relação as perguntas abertas contidas no questionário os participantes foram perguntados sobre o motivo da escolha da especialidade observamos muitas respostas acerca da maior afinidade como:

"Tive muita afinidade durante o curso."

"É a especialidade que mais me encantou e a mais desafiadora."

"É a especialidade que mais oferece raciocínio clínico."

E quanto ao motivo de terem escolhido o Hospital a resposta mais encontrada foi que este hospital é uma referência tanto em serviço como em profissionais de alta relevância dentro do campo escolhido como especialidade: 
"É uma instituição que eu confio."

"É considerado o melhor hospital com a melhor residência do estado."

"É o melhor corpo docente entre as residências do Pará."

A maioria dos médicos residentes revelou ser avaliado com provas teóricas e, algumas vezes em módulos específicos, com notas formativas, porém com critérios estabelecidos por cada preceptor e não de maneira uniforme, além de, muitas vezes, serem considerados critérios mais subjetivos. Destes, $68,4 \%$ não está satisfeita com este modo de avaliação. Dentre as formas que alguns residentes gostariam de ser avaliados observamos:

"Acredito que eu deveria ser avaliado de forma mais longitudinal e não de forma pontual."

"Gostaria de ser avaliado através de conceitos e não simplesmente com notas.

"Gostaria de incluir novos critérios de avaliação, pois prova teórica não avalia de forma holística."

Estas respostas corroboram com a pretensão desta pesquisa de avaliar a satisfação com as formas avaliativas atuais e o desejo do uso de ferramentas mais inovadoras e que levam a construção de conhecimento orientado por competências com uma avaliação formativa, porém com critérios mais objetivos e uniformes.

Seguramente a Residência Médica é um momento especial de preparação do médico para a vida prática profissional, onde, a partir deste momento, caminhará com as próprias pernas. Durante este período de aprendizado o seu conhecimento cresce de forma exponencial, treinando habilidades e criando o modo pessoal de trabalhar, com a ajuda dos preceptores, que passa a ser um modelo a ser seguido. Entretanto, neste período importante de formação as experiências podem ser angustiantes, quando não há alguém para discussão de casos, explicações acerca de conceitos ainda não conhecidos, muitas vezes o residente tomando para si a responsabilidade do cuidado com o paciente de forma integral (LAGOEIRO B, 2019).

Soma-se a isso o cansaço excessivo devido a longa jornada de trabalho dentro e fora da Residência médica, como observado nos relatos de alguns residentes que demonstraram cansaço após dias de serviço e plantões. Dentro desta discussão vale ainda ressaltar a importância da segurança do paciente, pois o estresse e o cansaço podem facilmente levar ao erro, já que todos somos humanos neste processo (LAGOEIRO B, 2019).

O fato de a grande maioria dos médicos residentes mostrarem-se insatisfeitos com seu processo avaliativo vai de encontro a vários estudos realizados nas mais diferentes profissões dentro da formação em saúde. Existem estudos em fonoaudiologia, fisioterapia, enfermagem, medicina (graduação e pós-graduação) que chegaram a resultados semelhantes a este estudo, ou seja, os estudantes ansiavam pelo uso de metodologias verdadeiramente ativas no sentido de levá-los a construir o próprio conhecimento de forma mais participativa, levando ao desenvolvimento do pensamento crítico, possibilitando a reflexão sobre o que é vivenciado e, a partir desta reflexão, poder solucionar problemas reais e atender as demandas da sociedade (LAGOEIRO B, 2019; WALTON J, et al., 2016).

Diante de discursos de médicos residentes preocupados com o bem-estar de seus pacientes, envolvidos em seus problemas e adquirindo para si suas angústias perante o caos na Saúde Pública, onde o residente está imerso, como linha de frente de atendimento pode-se perceber que o uso do Diário Reflexivo facilita este processo de crescimento pessoal e profissional, pois facilita a interação com professores e equipe multiprofissional, trazendo para o estudante a responsabilidade sobre seu aprendizado nesta forma de avaliar que leva em consideração os sentimentos e experiências vivenciadas na prática, em um ambiente seguro e com apoio emocional, como observado nos estudos de Lam R (2014), Buckley S, et al. (2009) e Sidebotham M (2018). O portfólio desenvolve aprendizagem mais profunda, melhora a capacidade reflexiva, com isso auxilia o aluno a enfrentar emoções, planejar trajetória de aprendizagem e construir confiança, além de facilitar o aprendizado, pois gerencia o grande volume de informações adquiridas e serve de catalisador para a 
aprendizagem de alta qualidade (MCEWEN LA, et al., 2015; MICHELS NRM, et al., 2016). Segundo Buckley S (2009), o portfólio melhora a capacidade de aprender de forma independente, a tomada de decisão, 0 pensamento crítico, aprimora habilidades de comunicação, autoconfiança, e a disposição para assumir responsabilidade sobre sua aprendizagem e profissionalismo (BUCKLEY S, et al., 2009).

Seu uso na educação vem crescendo consideravelmente nos últimos anos, seguindo uma tendência da formação crítica e reflexiva de profissionais de saúde, pois configura como uma ferramenta de avaliação distinta das metodologias tradicionais, que vai de encontro ao desejo dos residentes entrevistados nesta pesquisa, que demonstraram a vontade de serem avaliados de uma forma mais ativa na direção da construção do conhecimento a partir de competências clínicas, assim como no estudo de Padovani O \& Correa AK (2015). Alguns residentes sugeriram que o Diário Reflexivo poderia perder em confiabilidade e validade, assim como no estudo de Chang CC, et al. (2012), mas essa possível desvantagem poderia ser superada pela criação de critérios de avaliação mais objetivos e escolhidos em acordo comum entre todos os participantes. Outra desvantagem aventada também seria o não apoio institucional, porém isto seria remediado através de evidências científicas como o presente estudo, além de educação continuada (CHANG CC, et al., 2012).

$O$ fato de médicos residentes estarem insatisfeitos com seus métodos de avaliação nos leva a pensar que a criação de novas metodologias é extremamente necessária para não atrapalhar um processo tão importante e complexo como é o ensino-aprendizagem. Segundo Forte FDS (2016), com uso de ferramentas como o Diário Reflexivo pode-se adquirir alunos mais motivados e satisfeitos, pois são mais responsáveis e participativos. Este tipo de avaliação baseia-se em uma amostra mais ampla do trabalho do residente dentro do contexto vivenciado, mostrando suas necessidades e lacunas de conhecimento, suprindo o que for necessário, servindo de gatilho para mudanças na forma de avaliar e de pensar (FORTE FDS, 2016).

Dentre as limitações deste estudo encontra-se a amostra reduzida de participantes, porém não foi a intenção dos autores generalizar, mas sim obter uma compreensão mais profunda do assunto. As evidências do uso do portfólio na graduação médica ainda são limitadas, e mais ainda na pós-graduação, como é o caso da Residência Médica, especialmente porque o ato de refletir sobre as próprias ações com a intenção de intervir na realidade ainda é pouco difundido dentro da classe médica. $O$ uso de narrativas e o fato de falar sobre si mesmo também contam como dificuldades para médicos, o que também limita estudos que levam em consideração a reflexão como principal fator de avaliação., além do fato de que muitos residentes vêm de escolas médicas que ainda utilizam o método tradicional de ensino, aprendizagem e avaliação e, por isso, ainda há a dificuldade do uso de metodologias ativas e práticas.

\section{CONCLUSÃO}

Este estudo demonstrou a insatisfação da maioria dos médicos residentes quanto a metodologia avaliativa tradicional utilizada, requerendo novas metodologias, mais próximas da realidade neste contexto. O Diário Reflexivo permite o acompanhamento do processo de ensino-aprendizagem e motiva uma contínua reflexão podendo ser usado em diferentes cenários de aprendizagem, tanto em graduações, como em pósgraduações, como é o caso da Residência Médica, promovendo a emancipação progressiva do estudante, com aumento da interação entre professor e aluno dentro de um contexto pedagógico de aprendizagem significativa e esta ferramenta exige participação ativa dos alunos no processo de ensino e aprendizagem através da reflexão crítica da realidade. Ou seja, os médicos residentes vivenciam as experiências do dia-adia, e ao registrarem as atividades realizadas em diários alcançam a aprendizagem através da aquisição ade competências, conhecimentos e atitudes, além do desenvolvimento profissional, inerente a este momento de formação. É imprescindível ter em mente que o Diário Reflexivo não se trata apenas de acúmulo de papéis e documentos, mas sim de uma temática relevante e inovadora que busca beneficiar o desenvolvimento e crescimento do estudante em qualquer nível de ensino.

\section{REFERÊNCIAS}

1. BARDIN L. Análise de conteúdo. São Paulo: Edições 70, 2011.

2. BERNARDI MC, et al. Portfólio na avaliação do estudante de graduação na área da saúde: Estudo bibliométrico. Cogitare Enfermagem, Curitiba, 2015; 20 (1): 153-160. 
3. BUCKLEY S, et al. The educational effects of portfolios on undergraduate student learning: a Best Evidence Medical Education (BEME) systematic review BEME Guide No. 11. Med. Teach. 2009; 31: 282-298.

4. CARDOSO DAS, et al. Aprendizagem Reflexiva: o Uso do Portfólio Coletivo. Revista Brasileira de Educação Médica, [s.I.], 2015; 39 (3): 442-449.

5. CESARIO JB, et al. Portfólio reflexivo como estratégia de avaliação formativa. Rev Baiana Enferm, Salvador, 2016; 30 (1): 356-364.

6. CHANG C, et al. Reliability and validity of Web-based portfolio peer assessment: A case study for a senior high school's students taking computer course. Computers \& Education, [s.I.], 2011; 57: 1306-1316.

7. CHANG CC, et al. A comparative analysis of the consistency and difference among teacher-assessment, student self-assessment and peer-assessment in a Web-based portfolio assessment environment for high school students. Computers and Education, 2012; 58 (1): 303-320.

8. COTTA RMM, et al. Portfólios coletivos como método de ensino, aprendizagem e avaliação crítico-reflexiva: exercitando o aprender a conviver e a trabalhar junto. In: CONGRESSO IBERO-AMERICANO EM INVESTIGAÇÃO QUALITATIVA (CIAIQ), Anais... 2016.

9. COTTA RMM, et al. Portfólios crítico-reflexivos: uma proposta pedagógica centrada nas competências cognitivas e metacognitivas. Interface (Botucatu), Botucatu, 2015; 19 (54): 573-588.

10. FIGUEROA C, et al. Incorporación de paciente virtual en portafolio de estudiantes de medicina de pregrado. Rev Med Chile, 2015; 143: 175-82.

11. FORTE FDS, et al. Portfólio como estratégia de avaliação de estudantes de odontologia. Trabalho, Educação e Saúde, [s.l.], 2015; 3 (2): 25-38.

12. FORTE M, et al. Portfólio Reflexivo Eletrônico: Resultados de um Projeto Piloto. Rev. bras. educ. med., Rio de Janeiro, 2016; 40 (2): 234-244.

13. LAGOEIRO B. Você conhece os dramas da residência médica? Disponível em: https://pebmed.com.br/voceconhece-os-dramas-da-residencia-medica/ Acesso em: 23 jun 2019.

14. LAM R. Promoting self-regulated learning through portfolio assessment: Testimony and recommendations. Assessment and Evaluation in Higher Education, 2014; 39 (6): 699-714.

15. MCEWEN LA, et al. Developing and successfully implementing a competencybased portfolio assessment system in a postgraduate family medicine residency program. Acad Med. 2015; 90: 1515-1526.

16. MICHELS NRM, et al. Content validity of workplace-based portfolios: a multi-Centre study. Med Teach. 2016.

17. O'BRIEN CL, et al. Feasibility and Outcomes of Implementing a Portfolio Assessment System Alongside a Traditional Grading System. Academic Medicine, [s.I.], 2015; 91 (11): 1554-1560.

18. PADOVANI O, CORREA AK. Tecnologias no uso do portfólio reflexivo em cursos superiores na área da saúde, In: CONVIBRA, 2015, IV Congresso Online - Gestão, Educação e Promoção da Saúde, 2015.

19. SIDEBOTHAM M, et al. Preparing student midwives for professional practice: Evaluation of a student eportfolio assessment item. Nurse Education in Practice, 2018; 32: 84-89.

20. SOUZA RGS. Atributos Fundamentais dos Procedimentos de Avaliação. In: TIBERIO, I.F.C. et al. Avaliação prática de habilidades em medicina. São Paulo: Ed Atheneu, 2012, cap.1-11.

21. STELET BP, et al. Portfólio Reflexivo: subsídios filosóficos para uma práxis narrativa no ensino médico. Interface (Botucatu), 2017; 60: 165-176.

22. WALTON J, et a. Student ePortfolios to develop reflective skills and demonstrate competency development: Evaluation of a curriculum pilot project. European Journal of Dental Education; 2016; 20(2): 120-128.

23. WEBB TP, et al. Assessing Competency in Practice-Based Learning: A Foundation for Milestones in Learning Portfolio Entries. Journal Of Surgical Education, [s.I.], 2014; 71: 472-479. 